\title{
Link Error Prediction in Wireless Communication Systems with Quality Based Power Control
}

\author{
Wolfgang Karner, Olivia Nemethova, Markus Rupp \\ Institute of Communications and Radio-Frequency Engineering \\ Vienna University of Technology, Austria \\ Gusshausstrasse 25/389, A-1040 Vienna, Austria \\ Email: $\{$ wkarner, onemeth, mrupp $@$ nt.tuwien.ac.at
}

\begin{abstract}
The quality of end-to-end services over wireless communication networks highly depends on the error characteristics of the wireless links. By utilizing the link error characteristics for optimizing the higher layer protocols in a so called cross-layer approach, high quality improvements can be achieved, especially when considering the predictability of errors. In this document it is shown that wireless communication systems with quality based power control mechanisms entail such predictive nature of the link error characteristics. A method for obtaining estimated values of future link error probabilities in the UMTS (Universal Mobile Telecommunications System) DCH (Dedicated CHannel) as an example is presented in this document. Furthermore, it is shown that various well-known and often used error models based on Markov chains are not capable of representing the predictive nature of the channel, whereas a two-state alternating Weibull renewal process describes the characteristics properly.
\end{abstract}

\section{INTRODUCTION}

The overall goal in optimizing cellular wireless communication systems is to minimize the intra-system interference while increasing the QoS (Quality of Service) for the end user. Recent research has shown that the service quality can be improved considerably when optimizing higher layer protocols with awareness of the link error characteristics (e.g. [1]). Even higher gain can be reached when not only considering historical link error statistics but exploiting knowledge about the future, meaning explicitly using special characteristics of wireless links for predicting link errors ([2], [3]).

Many wireless networks - like the UMTS (Universal Mobile Telecommunications System) - try to minimize the interference in the system with a quality based power control algorithm by regulating the link power level according to the difference between the experienced and a target BER (Bit Error Ratio) or BLER (Block Error Ratio). A common way of implementing such a slow closed loop power control mechanism includes a sawtooth algorithm ( [4], [5]) which introduces periodicity in the link error characteristics as presented in this document. It will be shown that the periodicity of the quality based power control algorithm can be used to predict the link errors.

In scenarios with movement, the experienced link error characteristics heavily depend on time-variant fading effects. However, measurements in live UMTS networks [6] have shown that in static scenarios (meaning neither the mobile station, nor the base station are moving and there are also only few movements of persons or objects around the mobile station) the influence of the power control algorithm on the link error characteristics dominates over the fading effects. As there is an increasing demand of broadband wireless access via mobile communication networks for nomadic (fixed during their usage) mobile devices like notebooks, the static case becomes more and more important. The analysis in this work is based on measured link error traces of the UMTS DCH (Dedicated CHannel) in static scenarios in the city center of Vienna, Austria [6].

For the evaluation of the performance of new higher layer protocols or algorithms, measured link error traces often are not sufficient and models of the link error characteristics are required instead. Especially in the case when higher layer algorithms are exploiting the capability of predicting the link errors, the link error models have to represent the predictive nature of the channel properly. This work will show that well-known and widely used error models like a simple two-state Markov chain, Gilbert's model [7], the enhanced model of E.O. Elliot [8] and even the N-state partitioned Markov chains proposed by Fritchman [9] do not meet the requirements for that special purpose. On the other hand, the two-state alternating Weibull renewal process, as presented in [6], perfectly fits the observation.

This document is organized as follows. In Section II the impact of the quality based power control algorithm on the link error characteristics is shown. Section III presents the proposed method for predicting the resulting link errors and the achieved quality of error prediction is discussed. The poor capability of the above mentioned well-known classical link error models to represent the predictive nature of the channel is investigated in Section IV and Section V provides a summary and conclusions.

\section{LINK ERROR CHARACTERISTICS DUE TO QUALITY BASED POWER CONTROL}

In order to use radio resources in cellular wireless communication systems efficiently, the minimum transmit power for each link has to be found while meeting the quality target of the link. In the UMTS this task is performed by the OLPC (Outer Loop Power Control) algorithm [10] - a slow (100Hz) closed loop control mechanism which sets the target SIR (Signal to Interference Ratio) for the fast ILPC (Inner Loop Power 
Control) in order to converge to a required link quality, given by the network. The ILPC then regulates the link power level with a $1500 \mathrm{~Hz}$ rate to meet the target SIR.

Within 3GPP (3rd Generation Partnership Project) the OLPC is not entirely specified. A common way of implementing the control procedure is using CRC (Cyclic Redundancy Check) information feedback of the transmitted TBs (Transport Blocks) together with a sawtooth algorithm as described in [4] and [5]. If a TB was received in error, the target SIR value is increased by $K \Delta$, whereas in case of errorfree transmission it is reduced by $\Delta$, where $\Delta$ is the step size in $\mathrm{dB}$. If $K$ is chosen to be $1 / \mathrm{BLER}_{\text {target }}-1$, the algorithm tries to keep the BLER always less than or equal to BLER $_{\text {target }}=1 /(K+1)$. Thus, by selecting a proper value $K$ a quality level for the wireless link can be set.

Fig. 1 shows a schematic illustration of this OLPC algorithm for the case when an error occurs in the link with a probability

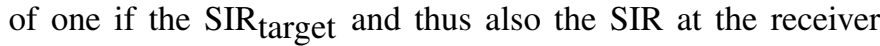
falls below a certain threshold. Obviously, the errors are easily predictable in this special case as there is an erroneous TB after $K$ error-free TBs.

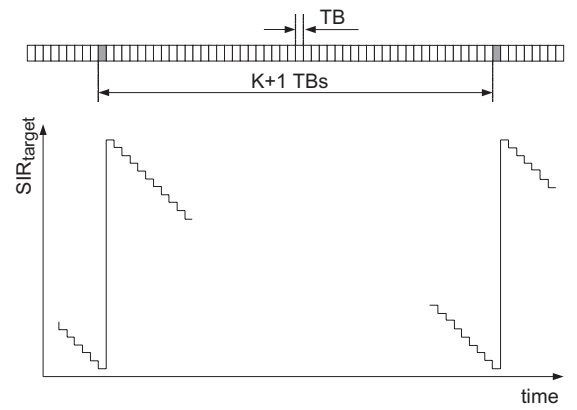

Fig. 1. Quality based power control (ideal).

In the UMTS DCH, burstiness is added to the error characteristics due to joint interleaving and coding over one TTI (Transmission Timing Interval) and also due to the stochastic nature of the channel. Fig. 2 shows a schematic illustration of the error bursts in the link which are spreading over one or several [11] subsequent TTIs. The power control algorithm, which is still trying to meet the quality target for the link, increases the target SIR by $K \Delta$ times the number of errors in the burst. This leads to a multiple of $K$ error-free TBs (long gap) between two error bursts.

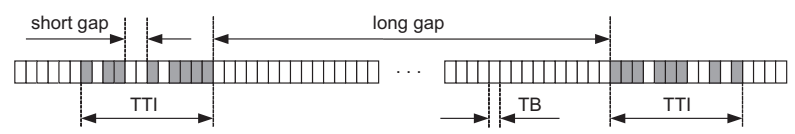

Fig. 2. Schematic illustration of long and short gaps.

Furthermore, the real channel of live UMTS networks especially in scenarios with movement - includes fading effects which may also provoke bursty error characteristics and link errors out of the OLPC cycle. However, measurements in live UMTS networks in the city center of Vienna, Austria have shown that the periodicity of the errors due to the OLPC dominates over the impact of the channel fading effects in static scenarios [6].
The measurements for the analysis in this work have been performed with a UMTS DCH in DL (Down Link) with 384kbit/s in RLC (Radio Link Control) AM (Acknowledged Mode) [12]. UDP data over IPv4, an RLC payload of 320 bits and 16 bits of CRC have been used. The TTI was $10 \mathrm{~ms}$ and there have been transmitted 12 TBs within each TTI by using a spreading factor of eight. The detailed description of the measurements is presented in [6].

In this document the following notation is used for describing the error process. An erroneously received TB is indicated by ' 1 ' while ' 0 ' means error-free transmission. A positive integer in the exponent determines the number of consecutive erroneous or error-free TBs (e.g. the sequence '000001' can be written as ' $0{ }^{5} 1$ ').

A gap with length $m$ is defined as the number of 0's between two 1's and

$$
f(m)=P\left(0^{m} 1 \mid 1\right)
$$

for all positive integers $m$ is the PMF (Probability Mass Function) of the gaplengths. The conditional probability $P(B \mid A)$ means the probability of sequence $B$, following sequence $A$. By definition,

$$
\sum_{m=0}^{\infty} P\left(0^{m} 1 \mid 1\right)=1
$$

and $P\left(0^{0} 1 \mid 1\right)=0$, as gaps with length zero are not considered for being gaplengths.

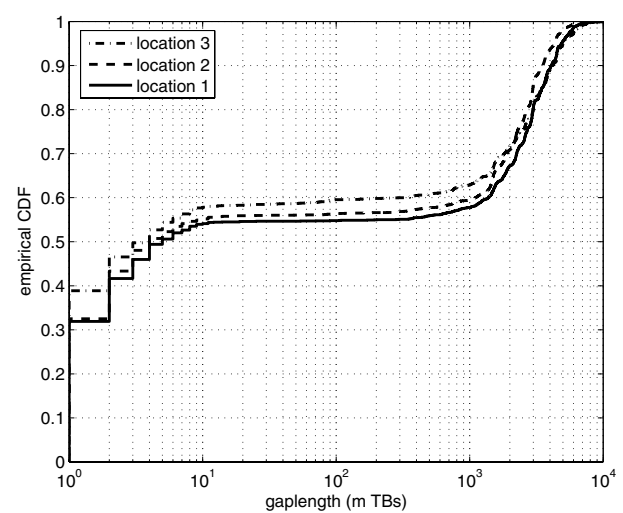

Fig. 3. Statistics of gaplengths based on measurements in static scenarios.

Fig. 3 shows measured results as empirical CDFs of the gaplengths

$$
F(m)=\mathrm{P}\{X \leq m\}=\sum_{k=0}^{m} P\left(0^{k} 1 \mid 1\right),
$$

where it can be observed that variations in gaplength occur in two different parts of the CDFs. There are the very short gaps $(\leq 12$ TBs) appearing within error bursts and the long gaps between the error bursts - a schematic illustration can also be seen in Fig. 2. Additionally, there is a third part in the CDFs between the short and the long gaps where there is only a very small probability for the appearance of gaps. This third part, together with the property of the distribution of the long gaps (the shape of the distribution is caused by the quality based power control algorithm) is used for the prediction of 
the errors in the link, as will be presented in the following section.

The measurements for Fig. 3 have been performed at different locations, dates and times of day in order to represent different propagation environments as well as different interference and network load situations. It can be observed that there is only little difference between the statistics. Furthermore, in measurements with additional DCH and also HSDPA users for creation of intra- and inter-cell interference we found out that additional interference in the DL does not change the measured statistics as long as the UMTS call admission control is working properly to keep the required link power off the power limit.

\section{LiNK ERROR PrEDICTION}

In order to be able to predict future link errors based on error statistics of the forward link, it is necessary to know about the past link errors at the transmitter side. For the UMTS DCH this can be achieved by utilizing the error feedback of the RLC AM with a variable maximum number of allowed retransmissions (zero would mean no retransmissions). The error feedback information is available at the transmitter a certain delay $\left(d_{F B}\right)$ after the transmission of the TB. Due to the fact that the feedback delay $\left(d_{F B}\right)$ is about the size of the length of short gaps, the error feedback information cannot be used to predict errors within error bursts. On the other hand, after waiting a time interval $d_{\text {min }}$, larger than the longest of the short gaps, without occurrence of reports of errors within this interval, it can be concluded that the current transmission takes place within one of the long error gaps and thus feedback information can be used for error prediction despite having feedback delay. A schematic illustration of the feedback delay and the proposed error prediction process is presented in Fig. 4.

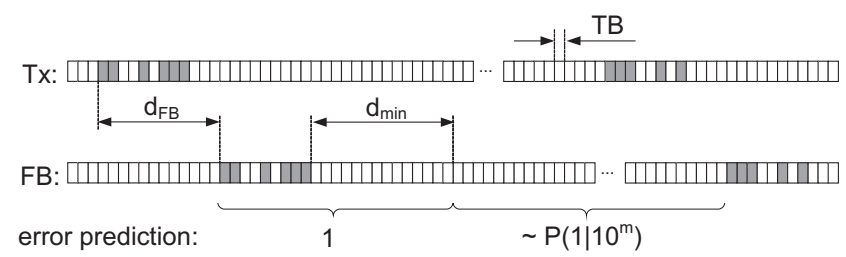

Fig. 4. Schematic illustration of error prediction.

For some applications like in [3] it is sufficient to detect the start of intervals with low error probability (the beginning of the long gaps after $d_{\text {min }}$ ), whereas higher sophisticated crosslayer methods [13] require more detailed information about the expected future error probability in the link.

As already mentioned, before the end of the error-free interval $d_{\text {min }}$, no error prediction is possible due to the feedback delay. Thus, beginning with the report of an erroneously received TB until the end of $d_{\text {min }}$, the prediction algorithm outputs an error probability of one for future transmitted TBs (like illustrated in Fig. 4). After $d_{\min }$, the error prediction starts using past statistics of the conditional link error probability $P\left(1 \mid 10^{m}\right)$ (the error probability conditioned to the number of error-free TBs since the last error). Statistics of $P\left(1 \mid 10^{m}\right)$ for a measured trace and for simulations with averaging over 1000 TBs in both cases, are shown in Fig. 5. Note that this averaging causes the conditional error probability larger than zero (instead of zero) at the error-free runlength of zero TBs.

From the measured statistics in Fig. 5 a linear behaviour of the conditional link error probability can already be anticipated, being more pronounced in the simulated curve which was created by using a link error model for obtaining more accurate statistics (weak statistics can be seen in the measured curve especially in the steep decline for error-free runlenghts of $>6500 \mathrm{TBs}$ ). The used link error model is based on a Markov modulated two-state alternating renewal process [6], with Weibull distributions for creating appropriate statistics of the short and the long gaps separately. In Section IV it is shown that this model is perfectly capable of representing the predictive nature of the link errors, whereas in contrast to that other well-known classical error models cannot be used for that special purpose. It will be presented in the following that the Weibull-like distribution of the long gaps causes the mentioned linear behaviour of the conditional error probability $P\left(1 \mid 10^{m}\right)$.

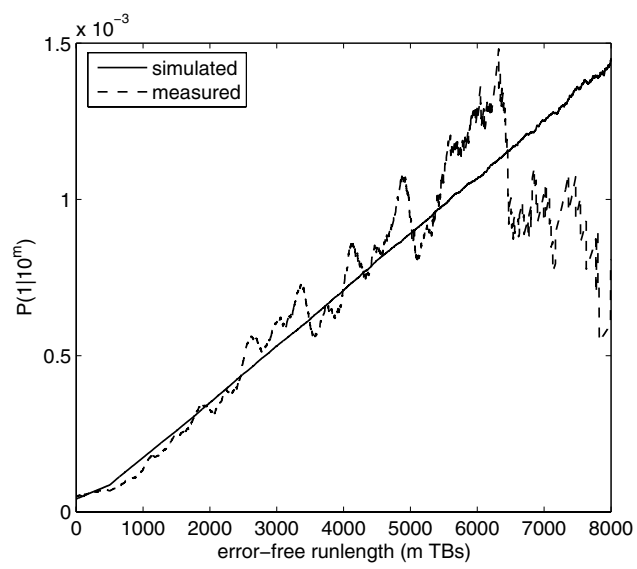

Fig. 5. Conditional link error probability, measured vs. simulated.

The conditional error probability (expected failure rate [28]) can be calculated by

$$
P\left(1 \mid 10^{m}\right)=\frac{f(m)}{1-F(m)}=\frac{P\left(0^{m} 1 \mid 1\right)}{P\left(0^{m} \mid 1\right)},
$$

where $P\left(0^{m} 1 \mid 1\right)$ is the PMF (Probability Mass Function) of the gaplengths and $P\left(0^{m} \mid 1\right)$ is their CCDF (Complementary Cumulative Distribution Function), which can also be written as

$$
P\left(0^{m} \mid 1\right)=1-F(m)=\sum_{k=m}^{\infty} P\left(0^{k} 1 \mid 1\right)
$$

and denotes the probability of having a gaplength of at least length $m$.

In [6] a link error model made up by a two-state alternating Weibull renewal process was presented. As in this model both the distribution of the short gaps and the distribution of the long gaps were perfectly fitted via a Weibull distribution, we 
insert the Weibull distribution in (4). The Weibull PDF and the Weibull CCDF [14] are given by

$$
\begin{gathered}
P\left(0^{m} 1 \mid 1\right)_{\text {Weibull }}=b a^{-b} m^{b-1} e^{-\left(\frac{m}{a}\right)^{b}}, \\
P\left(0^{m} \mid 1\right)_{\text {Weibull }}=e^{-\left(\frac{m}{a}\right)^{b}},
\end{gathered}
$$

respectively, and therefore (4) becomes

$$
P\left(1 \mid 10^{m}\right)_{W \text { eibull }}=\frac{b a^{-b} m^{b-1} e^{-\left(\frac{m}{a}\right)^{b}}}{e^{-\left(\frac{m}{a}\right)^{b}}}=b a^{-b} m^{b-1} \text {. }
$$

In [6] the parameter setting of the Weibull distribution for the long gaps (which is important for the error prediction in our case) was $a=3350$ for the scale parameter and $b=2.0176$ for the shape parameter in case of a static channel behaviour.

When considering the fact that the shape parameter $b$ is close to two we can take the conditional error probability $P\left(1 \mid 10^{m}\right)$ as linear increasing with $m$. (Note, a Weibull distribution with a shape parameter of two is equal to a Rayleigh distribution.)

As shown earlier, the information about errors in the forward link arrives at the transmitter after a certain feedback delay $\left(d_{F B}\right)$ which of course deteriorates the error prediction. In order to see the impact of $d_{F B}$ on the error prediction, in Fig. 6 the experienced error probability $P(1)$ is depicted over the estimated error probability $\hat{P}(1)=P\left(1 \mid 10^{m}\right)$ calculated from the feedback information for various $d_{F B}$ from zero up to $100 \mathrm{TBs}(=83 \mathrm{~ms})$. As expected, the accuracy of the error prediction gets worse with increasing feedback delay. While at a $d_{F B}$ of zero TBs the experienced error probability is equal to the predicted probability, with longer delay the experienced error probability becomes up to seven times higher. This is due to the fact that with increasing $d_{F B}$ the experienced errors in the forward link get more and more outside the interval where an error probability of one is predicted (right after the reception of an error message until the end of $d_{\min }$ ) and thus raising the experienced above the predicted error probability. Fig. 6 furthermore shows that for a feedback delay of 36 TBs ( $=3$ TTIs) or longer, the curves of experienced error probability are converging due to the fact that the longest error bursts are spreading over three TTIs [11] in static scenarios. Thus, for a $d_{F B} \geq 36$ the estimated error probability can be adjusted to be $\hat{P}(1)=\gamma P\left(1 \mid 10^{m}\right)$ with $\gamma=N_{e}-1 . N_{e}$ is the average number of erronous TBs within a burst $\left(N_{e}=8.1\right.$ in the static scenario), where a burst is considered as a group of errors containing short gaps.

In Fig. 7 the resulting quality of estimating future link error probabilities for a $d_{F B}$ of 36 TBs and $\gamma=7.1$ is presented. It can be observed that $|\hat{P}(1)-P(1)|$ is lower than $4 \cdot 10^{-4}$. We expect the estimation errors to be even smaller for simulations with longer error traces due to better statistics.

It can be seen that despite of having feedback delay, link error prediction is possible via statistics of past conditional error probabilities. By making use of the link error prediction capability together with the knowledge about the error probability in the link ( $0.267 \%$ in this case), cross-layer scheduling algorithms can improve data transmission over wireless links significantly [3] [13].

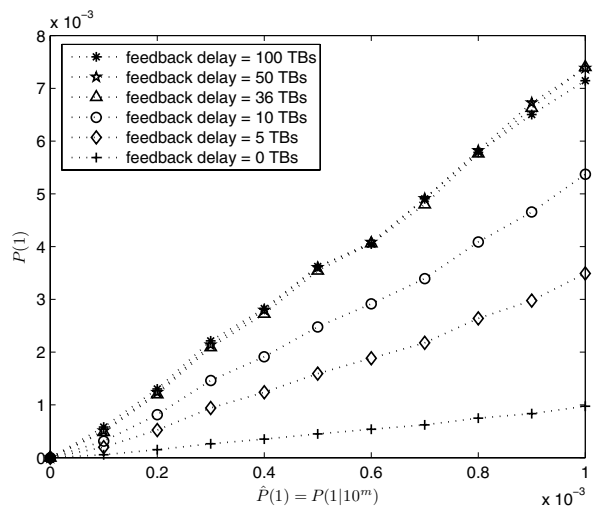

Fig. 6. Impact of feedback delay on the error prediction.

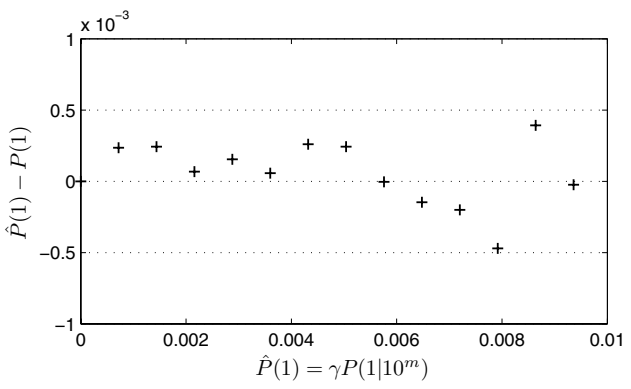

Fig. 7. Quality of error prediction.

\section{INFLUENCE OF LiNK ERROR MODELING ON ERROR PREDICTION}

For having sufficient statistics, often simulations with statistical link models are required instead of directly performing measurements or using simulations with measured traces. Beginning in the 1960s with the work of Gilbert [7], people understood that a memoryless BSC (Binary Symmetric Channel) is not capable of describing the bursty nature of communication channels. Gilbert's model is a two-state Markov model with one 'good' and one 'bad' state, where no errors occur in the 'good' state but in the 'bad' state the error probability takes some value $>0$. Enhancements to that model can be found in the work of Elliot [8] where errors can occur also in the 'good' state and published by Fritchman [9] who makes use of more than only one 'good' state. Up to now, these models are attracting lots of attention in publications, where researchers are using the models or try to improve the models' characteristics and usability [15]-[22]. Others are modeling the error characteristics by using further HMM (Hidden Markov Models) [23] [24] or even HSMM (Hidden Semi-Markov Models) [25], chaotic maps [26], deterministic generative models [27] and Markov modulated renewal processes [6] for meeting particular properties of the wireless links.

In the following, the well-known and often used models like the memoryless channel, the simplified Gilbert model (error probability equal to one in the 'bad' state) and Fritchman's partitioned Markov chains (whereof Gilbert's model is a special case) are compared to a model based on a two-state alternating Weibull renewal process [14] (published in [6]), 
regarding their capability to describe the predictive nature of wireless links with quality based power control.

In case of the memoryless channel (completely defined by the error probability) as well as the simplified Gilbert model (classical two-state Markov chain) which is well-defined by the error probability and the average burstlength (number of subsequently erroneously received blocks), the produced gaplengths (number of error-free blocks between two errors) follow a geometric distribution [28].

Calculating the failure rate $P\left(1 \mid 10^{m}\right)_{\text {geometric }}$ for geometric distributions according to (4)

$$
P\left(1 \mid 10^{m}\right)_{\text {geometric }}=\frac{p q^{m}}{\sum_{i=m}^{\infty} p q^{i}}=\frac{1}{\sum_{i=0}^{\infty} q^{i}}=\text { const, }
$$

we can see that the conditional error probability is constant independent of $m$ due to the memoryless property of the geometric distribution and thus, no error prediction by considering the error-free runlengths is possible.

When using Fritchman's partitioned Markov chains with two error-free states and following the proposal of [21] (estimating the model parameters by separate curve fitting for different parts of the distribution function), the measured distribution of gaplengths can be met like shown in Fig. 8. Note that with this configuration, Fritchman's model is equivalent to Gilbert's two-state model.

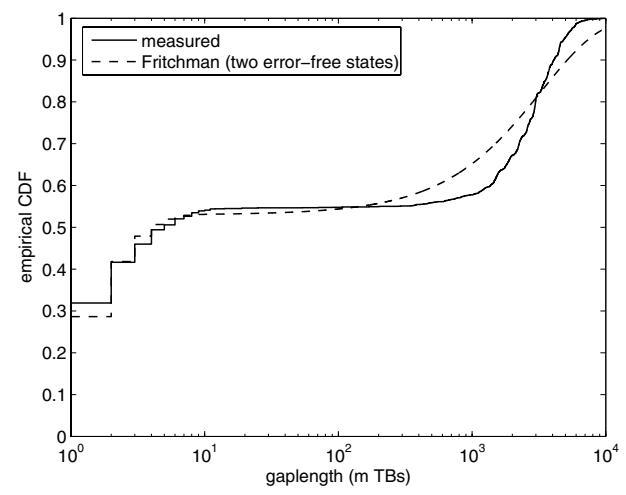

Fig. 8. Comparison of statistics of gaplengths, measured vs. Fritchman's model.

One of the error-free states is responsible for the distribution of the short gaps whereas the second state builds the form of the distribution for the long gaps. It can be shown that the fitting for the short gaps can be improved by adding more additional error-free states to the model but further states do not add accuracy to the fitting of the distribution for the long gaps. The reason for that can be seen in Fig. 9, where the empirical CDFs of the measured data, one Weibull and one geometric distributed CDF are shown in linear scale. Note that in Fig. 9 only the CDFs of the long gaplengths ( $>12$ TBs) are plotted, which are relevant for the error prediction in this work.

It can be observed that the measured data as well as the fitted Weibull curve are convex between zero and the inflection

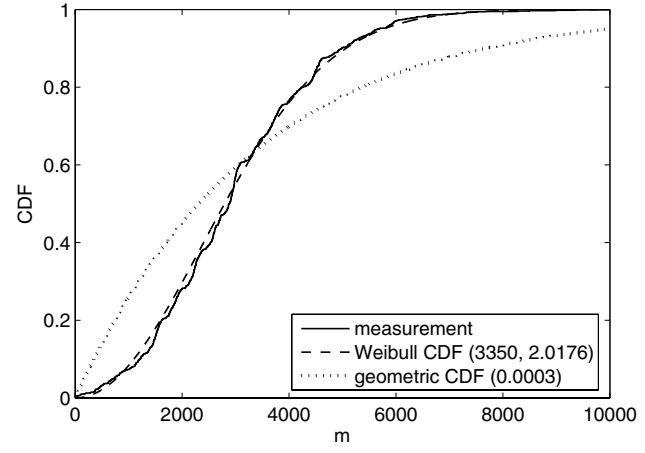

Fig. 9. Measured data vs. Weibull and geometric distribution.

point (mode $m_{0}$ ) given by

$$
m_{0}=\frac{(b-1)^{\frac{1}{b}}}{b^{\frac{1}{b}}} a,
$$

where $a$ and $b$ are scale and shape parameters of the Weibull distribution. The geometric distribution on the other hand is concave all over its support. Thus, perfect fitting of a geometric or polygeometric distribution (as formed by Fritchman's partitioned Markov chains) to the measured distribution is not possible. Note that the polygeometric $\mathrm{CDF}$ is given by

$$
F(m)_{\text {polygeometric }}=1-\sum_{i=1}^{N} \mu_{i} \lambda_{i}^{m},
$$

with the constraints of $0<\mu_{i}<1$ and $0<\lambda_{i}<1$ for Fritchman's partitioned Markov chains $\left(\mu_{i}\right.$ and $\lambda_{i}$ correspond to the transition probabilities of the Markov chain) and $N$ is the number of error-free states.

Furthermore, it can be concluded that the best fit is already reached with only one geometric distribution (meaning only one error-free state in Fritchman's model for the long gaps) due to the fact that every additional geometric distribution in a linear combination with only positive weighting factors adds concavity to the curve and thus increases the fitting error.

Therefore, Fritchman's as well as Gilbert's model (which is a special case of Fritchman's partitioned Markov chains) are also not capable of describing the predictive nature of the measured link error characteristics because of the constant failure rate $\left(P\left(1 \mid 10^{m}\right)_{\text {geometric }}=\right.$ const . $)$ due to the memoryless property of the geometric distribution. On the other hand, a link error model based on a two-state alternating Weibull renewal process [6] represents the measured statistics properly (see Fig. 10) and can perfectly be used in simulations with error prediction due to the increasing failure rate of Weibull distributions in case of shape parameter $b>1$, like shown in the previous section.

\section{SUMmary AND CONCLUSIONS}

In order to use radio resources as efficient as possible, many wireless communication systems are trying to minimize the link transmission power levels while still meeting the requirements for the quality of the services via a quality based power 


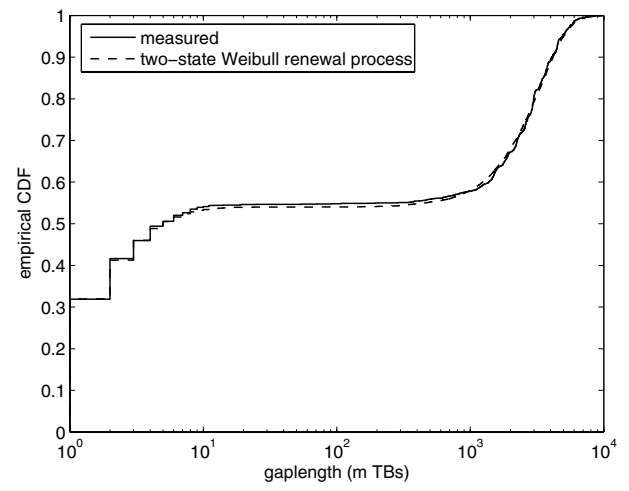

Fig. 10. Comparison of statistics of gaplengths, measured vs. simulated.

control algorithm. A common way of implementation makes use of a sawtooth algorithm which introduces periodicity to the link error characteristics. Measurements have shown that in static scenarios the influence of the power control algorithm on the link errors dominates over the fading effects and that this periodicity can be used to predict errors on the wireless link. Error prediction in turn can increase the gain out of cross-layer optimization methods and thus helps to improve the quality of service over wireless links significantly. It is shown in this document that the past statistics of conditional link error probabilities with a linear behaviour in case of the UMTS DCH can efficiently be used for error prediction.

For the performance evaluation of higher layer protocols or algorithms often models of the link error characteristics are required. In this work we are comparing the capability of several well-known link error models to describe the predictive nature of wireless links with quality based power control. It was found that the models based on geometric or polygeometric distributions like a classic two-state Markov chain, Gilbert's model or Fritchman's partitioned Markov chains are not appropriate for simulating error predictions due to the constant failure rate of the geometric distribution. On the other hand, a two-state alternating renewal process including Weibull distributions with increasing failure rate, perfectly meets the requirements for modeling the predictive nature of the wireless links.

\section{ACKNOWLEDGEMENTS}

We thank mobilkom austria AG for technical and financial support of this work. The views expressed in this paper are those of the authors and do not necessarily reflect the views within mobilkom austria AG.

\section{REFERENCES}

[1] O. Nemethova, W. Karner, A. Al-Moghrabi, M. Rupp, "Cross-Layer Error Detection for H.264 Video over UMTS," in Proc. of Wireless Personal Multimedia Communications 2005 (WPMC 2005), Aalborg, Denmark, Sept. 2005.

[2] P. Koutsakis, "Scheduling and Call Admission Control for Burst-Error Wireless Channels," 10th IEEE Symposium on Computers and Communications (ISCC 2005), 2005.
[3] W. Karner, O. Nemethova, P. Svoboda, M. Rupp, "Link Error Prediction Based Cross-Layer Scheduling for Video Streaming over UMTS," in Proc. of the 15th IST Mobile \& Wireless Communications Summit 2006, Myconos, Greece, June 2006.

[4] H. Holma and A. Toskala, WCDMA for UMTS, Radio Access For Third Generation Mobile Communications, John Wiley \& Sons, Ltd., 2004.

[5] A. Sampath, P.S. Kumar, J.M. Holtzman, "On Setting Reverse Link Target SIR in a CDMA System," 47th IEEE Vehicular Technology Conference, vol.2, pp. 929-933, 1997.

[6] W. Karner, M. Rupp, "Measurement based Analysis and Modelling of UMTS DCH Error Characteristics for Static Scenarios," in Proc. of 8th International Symposium on DSP and Communication Systems (DSPCS'2005), Sunshine Coast, Australia, Dec. 2005.

[7] E.N. Gilbert, "Capacity of a burst-noise channel," Bell Systems Technical Journal, vol. 39, pp. 1253-1265, Sept. 1960.

[8] E.O. Elliot, "Estimates of error rates for codes on burst-noise channels," Bell Systems Technical Journal, vol. 42, pp. 1977-1997, Sept. 1963.

[9] B.D. Fritchman, "A Binary Channel Characterization Using Partitioned Markov Chains," IEEE Trans. Information Theory, vol. 13, no. 2, pp. 221-227, Apr. 1967.

[10] 3GPP TR 25.101, "User Equipment (UE) Radio Transmission and Reception (FDD)," v.6.12.0, June 2006.

[11] W. Karner, P. Svoboda, M. Rupp, "A UMTS DL DCH Error Model Based on Measurements in Live Networks," in Proc. 12th International Conference on Telecommunications (ICT 2005), Capetown, South Africa, May 2005.

[12] 3GPP TR 25.322, "Radio Link Control (RLC) Protocol Specification," v.4.12.0, June 2004.

[13] O. Nemethova, W. Karner, M. Rupp, "Error Prediction Based Redundancy Control for Robust Transmission of Video over Wireless Links," IEEE Int. Conf. on Communications (ICC 2007), Glasgow, Scotland, June 2007.

[14] D.N.P. Murthy, M. Xie, R. Jiang, Weibull Models, John Wiley \& Sons, Ltd., 2004.

[15] J-P.A. Adoul, B.D. Fritchman, "A Critical Statistic for Channels With Memory," IEEE Trans. on Information Theory, vol. 18, no. 1, Jan. 1972.

[16] C. Pimentel, F. Blake, "Modeling Burst Channels Using Partitioned Fritchman's Markov Models," IEEE Transactions on Vehicular Technology, vol. 47, no. 3, Aug. 1998.

[17] C. Pimentel, F. Blake, "Enumeration of Markov Chains and Burst Error Statistics for Finite State Channel Models," IEEE Transactions on Vehicular Technology, vol. 48, no. 2, March 1999.

[18] L.N. Kanal, A.R.K. Sastry, "Models for Channels with Memory and Their Applications to Error Control," Proceedings of the IEEE, vol. 66, no. 7, July 1978.

[19] W. Turin, M.M. Sondhi, "Modeling Error Sources in Digital Channels," IEEE Journal on Selected Areas in Communications, vol. 11, no. 3, April 1993.

[20] S. Sivaprakasam, K.S. Shanmugan, "An Equivalent Markov Model for Burst Errors in Digital Channels," IEEE Transactions on Communications, vol. 43, no.2/3/4, 1995.

[21] J-Y. Chouinard, M. Lecours, G.Y. Delisle, "Estimation of Gilbert's and Fritchman's Models Parameters Using the Gradient Method for Digital Mobile Radio Channels," IEEE Transactions on Vehicular Technology, vol. 37, no. 3, Aug. 1988.

[22] V. Tralli, M. Zorzi, "Markov Models for the Physical Layer Block Error Process in a WCDMA Cellular System," IEEE GLOBECOM 2002, vol. 2, pp. 1925-1929, Nov. 2002.

[23] W. Turin, Digital Transmission Systems: Performance Analysis and Modelling. New York: McGraw-Hill, 1999.

[24] L.R. Rabiner, B.H. Juang, "An Introduction to Hidden Markov Models," IEEE ASSP Magazine, vol. 3, pp. 4-16, Jan. 1986.

[25] M. Azimi, P. Nasiopoulos, R.K. Ward, "Offline and Online Identification of Hidden Semi-Markov Models," IEEE Transactions on Signal Processing, vol. 53, no. 8, Aug. 2005.

[26] A. Köpke, A. Willig, H. Karl, "Chaotic Maps as Parsimonious Bit Error Models of Wireless Channels," IEEE INFOCOM 2003, vol. 1, pp. 513523, April 2003

[27] C.X. Wang, W. Xu, "Packet-Level Error Models for Digital Wireless Channels," IEEE Int. Conf. on Communications (ICC 2005), vol. 4, pp. 2184-2189, May 2005.

[28] A. Papoulis, P.S. Unnikrishna, Probability, Random Variables, and Stochastic Processes, McGraw-Hill, 2002. 